\title{
Optimisation des structures en interaction fluide-structure
}

\section{Structural optimization in fluid-structure interaction}

\author{
R. El Maani ${ }^{1}$, B. Radi ${ }^{2}$, A. El Hami ${ }^{3}$ \\ 1'LOFIMS, INSA de Rouen, France, rabii.el_maani@insa-rouen.fr \\ ${ }^{2}$ LIMII, FST Settat, Maroc, bouchaib.radi@yahoo.fr \\ ${ }^{3}$ LOFIMS, INSA de Rouen, France, abdelkhalak.elhami@insa-rouen.fr
}

RÉSUMÉ. Les problèmes d'optimisation des structures élastiques sous écoulement de fluide se posent souvent dans de nombreuses applications qui constituent un problème multi-physique particulièrement important. Cet article présente des applications générales et efficaces pour l'analyse d'optimisation structurale de systèmes caractérisés par des problèmes d'interaction fluide-structure (IFS) fortement couplés. L'optimisation de conception, de forme et topologique ont été étudiées dans ce cas et les résultats obtenus ont été ensuite présentés et discutés. Dans les problèmes d'IFS, la structure peut présenter de grandes déformations en raison de l'interaction avec le domaine fluide, ce qui entraîne un comportement structurel non linéaire de géométrie et des conditions d'interface de couplage non linéaires. Les simulations des problèmes d'interaction fluide-structure à optimiser ont été analysées avec les solveurs ANSYS/ Mechanical et ANSYS/Flotran pour le calcul de la dynamique des fluides.

ABSTRACT. Structural optimization problems for elastic structures in flows arise frequently in many applications that form a particularly important of multi-physics problems. This paper presents general and efficient applications for structural optimization analysis of systems characterized as strongly coupled fluid-structure interaction (FSI) problems. Design, shape and topological optimization were studied in thi $s$ case and the obtained results were then presented and discussed. In FSI problems, due to the interaction with the fluid domain, resulting in geometrically nonlinear structural behaviour and nonlinear interface coupling conditions. The fluid-structure interaction simulations were traited using ANSYS/Mechanical and ANSYS/Flotran for fluid computational dynamics.

MOTS-CLÉS. Interaction fluide-structure, optimisation de conception, optimisation de forme, optimisation topologique. KEYWORDS. Fluid-structure interaction, design optimization, shape optimization, topological optimization.

\section{Introduction}

Déterminer la forme appropriée des composants structuraux est un problème de première importance pour l'ingénieur. Dans tous les domaines de la mécanique des structures, l'impact de la bonne conception d'une pièce est très important sur sa résistance, sa durée de vie et son utilisation en service. Ce défi est quotidien dans les secteurs de pointe tels que la recherche spatiale, l'aéronautique, l'automobile, la construction navale, la mécanique de précision et les ouvrages d'art en génie civil. Le développement de l'art de l'ingénieur requiert des efforts considérables pour améliorer sans cesse les techniques de conception des structures. L'optimisation intervient de façon primordiale dans l'augmentation des performances et la réduction de la masse des engins aérospatiaux et automobiles, entrânant ainsi de substantielles économies d'énergie.

Le développement constant des techniques de Conception Assistée par Ordinateur (CAO) et des stratégies d'optimisation s'inscrit dans ce cadre. L'optimisation des structures soulève depuis plus de vingt ans le plus vif intérêt. Encore trop peu appliquée aux techniques classiques de bureau d'études, elle s'y intègre progressivement au fur et à mesure que s'accroît sa robustesse. Parti des problèmes les plus simples, le champ d'application de l'optimisation structurale s'étend aujourd'hui à de nouveaux défis toujours plus intéressants (El Hami A., and Radi B., 2011, Craveur J. C., Bruyneel M., and Gourmelen P., 2014). 
Pour illustrer les techniques d'optimisation structurale, on peut scinder arbitrairement l'optimisation des structures en trois grandes familles. L'optimisation de dimensionnement où les variables sont des sections, des épaisseurs ou toutes quantités qui peuvent évoluer sans nécessiter de modification du maillage en éléments finis et la forme étant fixée. L'Optimisation de forme admettant des changements de forme compatible avec une topologie fixée au préalable et finalement l'optimisation topologique qui permet de modifier plus fondamentalement la nature de la structure.

Dans cet article, l'optimisation structurale des problèmes tenant compte de l'interaction fluide-structure sera présentée pour le cas de l'optimisation de conception de forme et topologique avec différentes applications, et les résultats obtenus pour les trois cas seront ensuite présentés et discutés. Pour les simulations IFS, une approche implicite paritionnée a été employée, qui utilise une formulation Lagrangienne pour la structure et une formulation Eulerienne pour le fluide (Souli M., and Benson D. J., 2010, ANSYS 2012, Huang S., Li R., and Li Q. S., 2013).

\section{Problème d'interaction fluide-structure}

Un problème général d'interaction fluide-structure consiste en la description des champs solide et fluide, des conditions de l'interface conjointe et des conditions aux limites restantes. Dans cet article, on se limite à des écoulements incompressibles, ce qui est un choix raisonnable pour de nombreuses applications d'ingénierie.

\section{1. Équations pour le fluide}

La partie fluide du problème IFS est régie par les équations de Navier-Stokes pour un écoulement incompressible qui peuvent être écrites sur le domaine spatial de la mécanique des fluides comme (Patankar S. V., 1980, Ferziger J. H., and Peric M., 2002) :

$$
\begin{aligned}
\frac{(\partial v)}{\partial t}+\nabla \cdot(\rho v \otimes v-\sigma)-\rho f & =0 \\
\nabla \cdot v & =0
\end{aligned}
$$

où $\rho, v$, et $f$ sont la densité, la vitesse et la force externe, et $\sigma$ le tenseur des contraintes.

\section{2. Équations pour la structure}

L'équation régissant la réponse dynamique d'une structure ou d'un milieu élastique peut être écrite sous forme matricielle (Chopra A., 2001) comme :

$$
m \ddot{u}+c \dot{u}+k u=f(t)
$$

où $m$ est la matrice masse de la structure, $\ddot{u}$ le vecteur d'accélération, $c$ la matrice d'amortissement de la structure, $\dot{u}$ le vecteur vitesse, $k$ la matrice de rigidité de la structure, $u$ le vecteur déplacement et $f$ le vecteur des forces en fonction du temps. 


\subsection{Conditions d'interface}

Les conditions principales à l'interface sont les conditions de couplage dynamique et cinématique. L'équilibre des forces exige l'égalité des vecteurs de contraintes comme suit :

$$
\sigma^{f} . n=\sigma^{s} . n \quad \forall x \in \Gamma^{f s i}
$$

aussi l'égalité des vitesses normales à l'interface :

$$
v . n=\frac{\partial u}{\partial t} . n \quad \forall x \in \Gamma^{f s i}
$$

\section{Forme mathématique générale du problème d'optimisation}

Un problème général d'optimisation des structures prend la forme :

$$
\left\{\begin{array}{l}
\text { minimize } f(x, y) \text { with respect to } x \text { and } y \\
\text { subject to }\left\{\begin{array}{l}
\text { contraintes de comportement sur } y \\
\text { contraintes de conception sur } x
\end{array}\right.
\end{array}\right.
$$

Deux types de contraintes sont indiqués dans [5] : (1) Les contraintes de comportement sont des contraintes sur la variable d'état $y$. Généralement, ils sont écrits $g(y) \leq 0$, où $g$ est une fonction qui représente, par exemple, un déplacement dans une certaine direction. (2) Les contraintes de conception sont des contraintes similaires impliquant la variable de conception $x$ (Christensen P. W., and Klarbring A., 2009).

\section{Simulation numérique}

\subsection{Optimisation de conception d'une aile d'avion}

L'exemple considéré est une aile d'avion de section symétrique et de longueur de $2 \mathrm{~m}$ comme illustrée sur la figure 1.

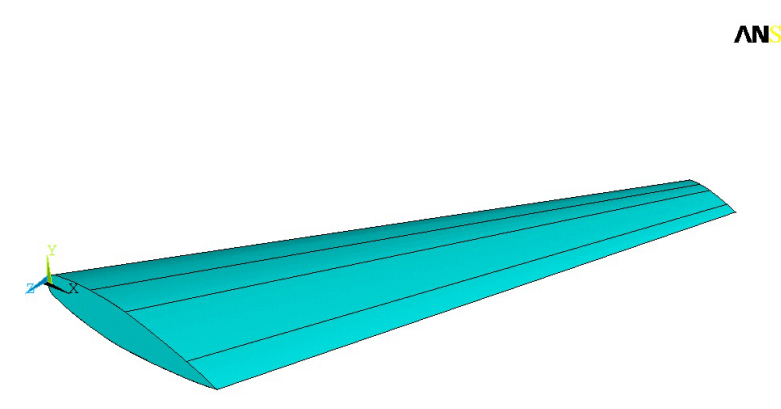

Figure 1. Aile d'avion à optimiser

L'objectif dans le cas suivant est de minimiser le volume de l'aile d'avion sous une contrainte fréquentielle. Les caractéristiques mécaniques de la structure sont les suivants : module d'Young $E=7.1 e 10$ $\mathrm{N} / \mathrm{m}^{2}$, cfficient de Poisson $\nu=0.3$ et la densité $\rho=2770 \mathrm{~kg} / \mathrm{m}^{3}$. 
Les variables de conception sont les dimensions de la section droite $A_{0}, B_{0}, C_{0}$, et $D_{0}$, illustrées sur le tableau 4.1.

\begin{tabular}{|c|c|c|c|c|}
\hline Variables & $A_{0}$ & $B_{0}$ & $C_{0}$ & $D_{0}$ \\
\hline Dimensions (m) & 0.054 & 0.092 & 0.096 & 0.044 \\
\hline
\end{tabular}

Tableau 4.1. Paramétrisation de l'aile

Le tableau 4.2 montre les résultats des valeurs optimales calculées pour un dimensionnement optimisé afin de satisfaire la contrainte fréquentielle imposée.

\begin{tabular}{|c|c|c|}
\hline Paramètres & Valeur initiale & Valeur optimale \\
\hline$A_{0}$ & 0.054 & 0.035485 \\
$B_{0}$ & 0.092 & 0.080140 \\
$C_{0}$ & 0.096 & 0.092598 \\
$D_{0}$ & 0.044 & 0.035341 \\
$\mathrm{~V}$ & 0.148595 & 0.134715 \\
$f_{1}$ & 31.321 & 29.072 \\
\hline
\end{tabular}

Tableau 4.2. Résultats d'optimisation

\subsection{Optimisation de forme d'une plaque}

Comme cas de test, nous considérons une plaque élastique en 2D soumise aux forces d'un écoulement de fluide dans un canal avec une vitesse d'entrée de $0.68 \mathrm{~m} / \mathrm{s}$. La géométrie du problème est représenté sur la figure 2.

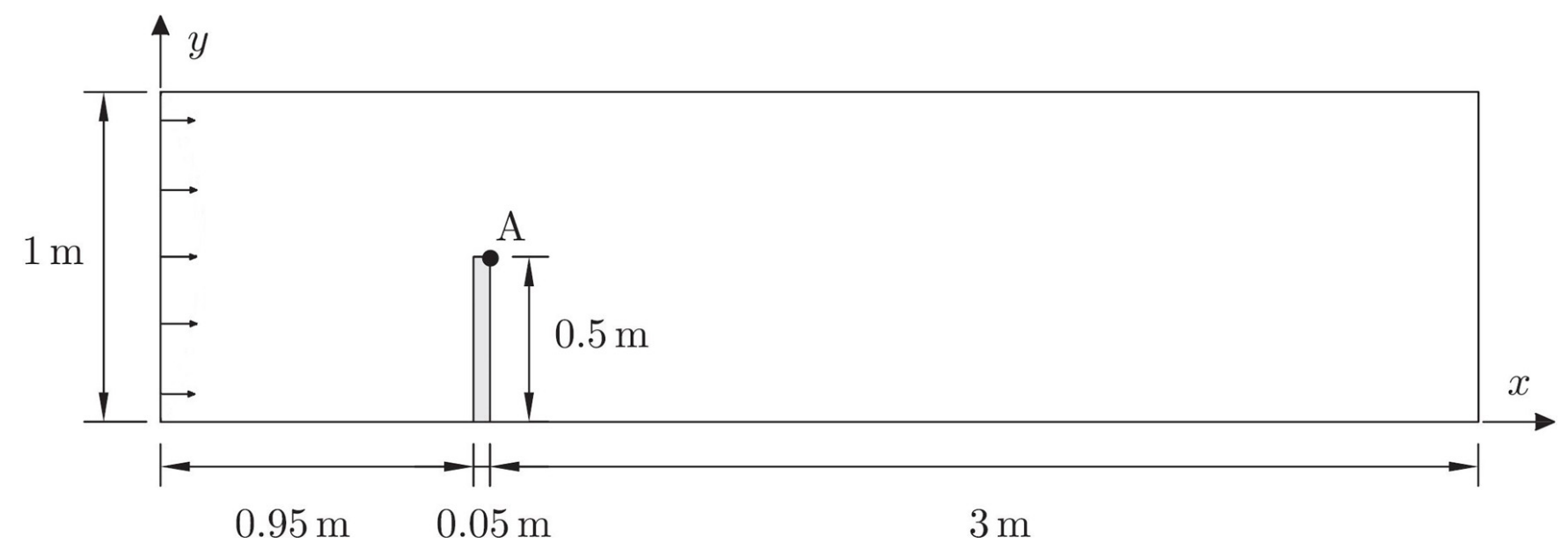

Figure 2. Géométrie et conditions aux limites de la plaque

À la sortie, une condition de gradient nul est appliqué. Aux parois du canal et de la plaque, une condition de non-glissement est employé. Les paramètres des matériaux appropriés pour le fluide et la structure sont les suivants : $\rho_{f}=1000 \mathrm{~kg} / \mathrm{m}^{3}, \mu_{f}=1 \mathrm{~kg} / \mathrm{m} . \mathrm{s}, E_{s}=5000 \mathrm{~N} / \mathrm{m}^{2}$ et $\nu_{s}=0.4$. 


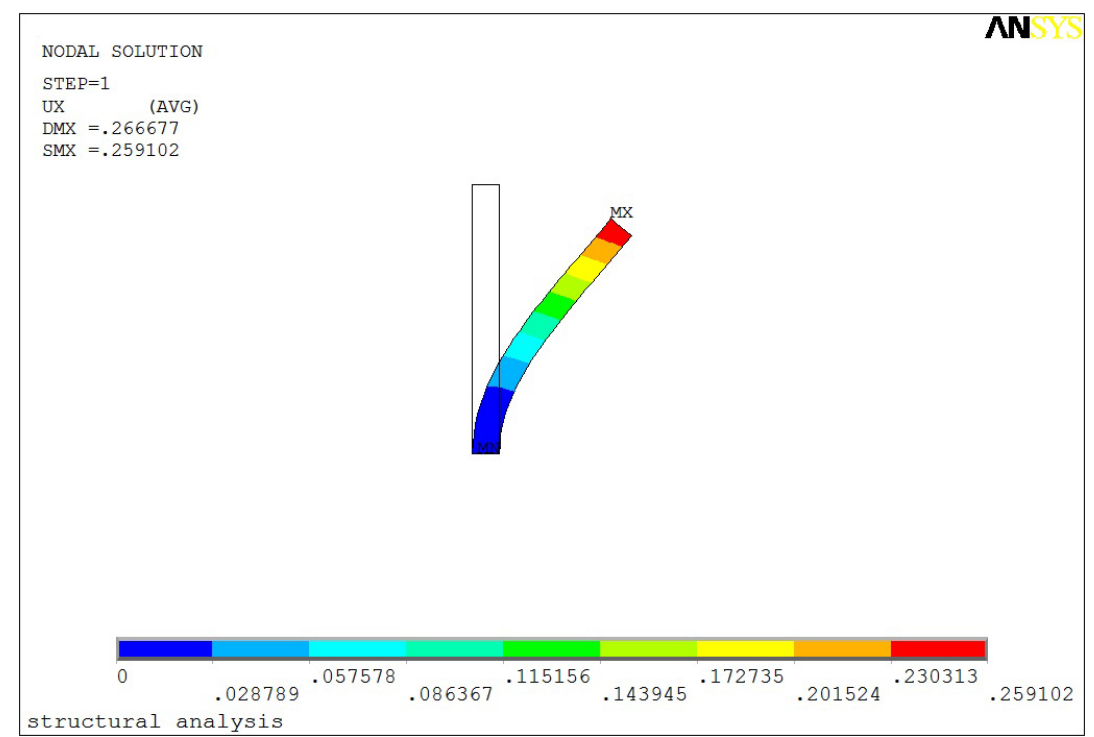

Figure 3. Déflection initiale de la plaque

L'objectif dans ce cadre d'optimisation de forme est d'obtenir une déflection minimale de la plaque (par rapport à la déflection initiale sur la figure 3) avec un volume minimal.

Le tableau 4.3 affiche la répartition du déplacement pour la forme optimisée à l'état déformé pour le cas où la contrainte est un volume minimale ou maximale obtenue avec la simulation IFS. Ces calculs conduisent à des résultats d'optimisation avec une déflection minimale de la plaque $u_{X}^{A}=1.67 \mathrm{~m}$.

\begin{tabular}{|c|c|c|}
\hline Paramètres & Valeur initiale & Valeur optimale \\
\hline$d_{1}$ & 0.95 & 0.94513 \\
$d_{2}$ & 0.95 & 0.94075 \\
$d_{3}$ & 0.95 & 0.97476 \\
$d_{4}$ & 1 & 0.98490 \\
$d_{5}$ & 1 & 1.0217 \\
Vol. & 0.025 & 0.023205 \\
Dépl. & 0.259 & 0.167
\end{tabular}

Tableau 4.3. Résultats d'optimisation

\subsection{Optimisation topologique d'un profil d'aile}

Soit un profil d'aile d'avion comme illustré par la figure 4, le but dans ce cas d'optimisation topologique est de trouver la meilleure utilisation du matériel pour ce profil de telle sorte que le critère objectif (la fréquence propre) prend une valeur maximale sous réserve d'une contrainte de réduction de volume.

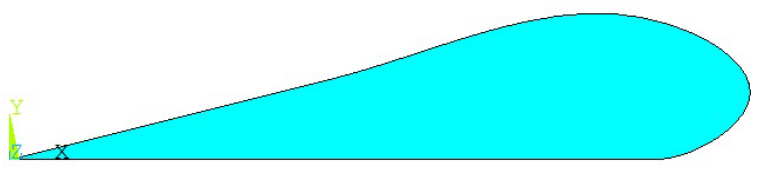

Figure 4. Profil d'aile à optimiser topologiquement 
Les propriétés des matériaux utilisées pour ce cas sont données comme suit :

Module d'Young $(E)=2.11 E 10 \mathrm{~N} / \mathrm{m}^{2}$

Coefficient de Poisson $(\nu)=0.3$

Densité $(\rho)=2770 \mathrm{~kg} / \mathrm{m}^{3}$

Le problème de l'optimisation peut s'écrire sous la forme :

$$
\left\{\begin{aligned}
\min : & V \\
\text { s.c }: & f_{1} \geq f
\end{aligned}\right.
$$

Les résultats de l'optimisation topologique sont présentés sur les figures 5, les surfaces en bleu désignent la matière qui peut être enlevée et qui est souvent remplacée par les concepteurs par des vides de formes circulaires ou elliptiques, le tableau 4.4 donne leurs valeurs optimales résultantes.

\begin{tabular}{|c|c|c|}
\hline & Valeur initiale & Valeur calculée \\
\hline Volume & 0.195262 & 0.195213 \\
\hline Freq1 & 631.719 & 675.333 \\
\hline
\end{tabular}

Tableau 4.4. Résultats d'optimisation
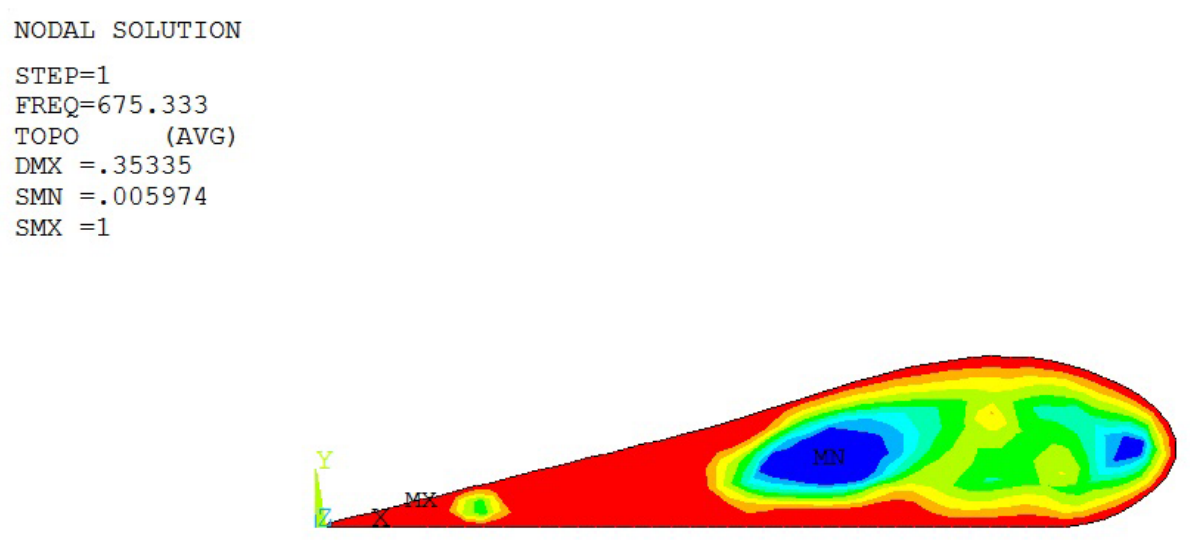

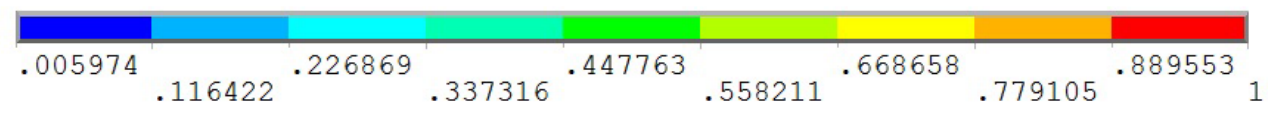

Figure 5. Profil d'aile à optimiser

\section{Conclusion}

Les problèmes d'optimisation des structures en interaction avec un fluide en écoulement ont été discutés dans cet article. Une forme mathématique générale de l'optimisation et des problèmes d'interaction fluide-structure a été brièvement présentée avec leurs équations de base. On a d'abord commencé par 
l'optimisation de conception d'une aile d'avion soumise à des charges aérodynamiques, puis une optimisation de forme d'une plaque élastique soumise à un écoulement de fluide et enfin une optimisation topologique d'un profil d'aile d'avion en comparant les valeurs initiales et optimisées et les formes résultantes pour les trois cas d'optimisation.

\section{Remerciements}

Les auteurs tiennent à remercier "Erasmus Mundus Programme, Action 2 - STRAND 1, Lot 1, North Africa Countries" pour leur soutien financier pour la réalisation de ce travail.

\section{Bibliographie}

El HAmi A., and RADi B., Fiabilité and optimisation des systèmes, Ellipses, 2011.

Craveur J. C., Bruyneel M., and Gourmelen P., Optimisation des structures mécaniques : Méthodes numériques et éléments finis, Dunod, 2014.

Souli M., and Benson D. J., Arbitrary Lagrangian-Eulerian and Fluid-Structure Interaction, ISTE Ltd and John Wiley Sons, 2010.

ANSYS. Complete User's Manual Set : ANSYS Theory Reference 14.0, ANSYS, INC., Canonsburg, USA, 2012.

PATANKAR S. V., Numerical Heat Transfer and Fluid Flow, Hemisphere Publishing, New York, USA, 1980.

Ferziger J. H., and PERIC M., Computational Methods for Fluid Dynamics, Springer, Berlin, Germany, 2002.

Chopra A., Dynamics of Structures, Pearson Prentice Hall, 2nd ed., 2001.

YUN Z., and HuI Y., Coupled fluid structure flutter analysis of a transonic fan, Chinese Journal of Aeronautics, 24, 258-264, 2011.

HUANG S., Li R., and LI Q. S., Numerical simulation on fluid-structure interaction of wind around super-tall building at high reynolds number conditions. Structural Engineering and Mechanics, An Int'l Journal, 46(2), 197-212, 2013.

Christensen P. W., and Klarbring A., An introduction to structural optimization, Springer, 2009. 